\title{
Applications of Artificial Intelligence in Machine Learning: Review and Prospect
}

\author{
Sumit Das \\ Department of IT \\ JIS College of \\ Engineering, Kalyani, \\ India
}

\author{
Aritra Dey \\ Department of EE \\ JIS College of \\ Engineering, Kalyani, \\ India
}

\author{
Akash Pal \\ Department of CSE \\ JIS College of \\ Engineering, Kalyani, \\ India
}

\author{
Nabamita Roy \\ Department of CSE \\ JIS College of \\ Engineering, Kalyani, \\ India
}

\begin{abstract}
Machine learning is one of the most exciting recent technologies in Artificial Intelligence. Learning algorithms in many applications that's we make use of daily. Every time a web search engine like Google or Bing is used to search the internet, one of the reasons that works so well is because a learning algorithm, one implemented by Google or Microsoft, has learned how to rank web pages. Every time Facebook is used and it recognizes friends' photos, that's also machine learning. Spam filters in email saves the user from having to wade through tons of spam email, that's also a learning algorithm. In this paper, a brief review and future prospect of the vast applications of machine learning has been made.
\end{abstract}

\section{Keywords}

Artificial intelligence, Machine learning, Supervised learning, Unsupervised learning, Reinforcement learning Applications.

\section{INTRODUCTION}

An Artificial Intelligence (AI) program is called Intelligent Agent. Intelligent agent gets to interact with the environment. The agent can identify the state of an environment through its sensors and then it can affect the state through its actuators.

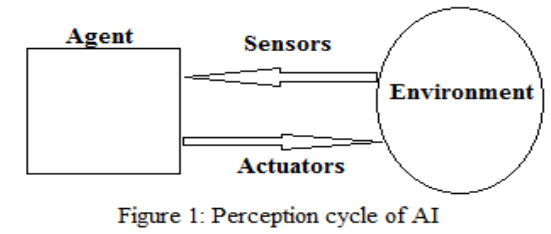

The important aspect of AI is the control policy of the agent which implies how the inputs obtained from the sensors are translated to the actuators, in other words how the sensors are mapped to the actuators, this is made possible by a function within the agent.

The ultimate goal of AI is to develop human like intelligence in machines. However such a dream can be accomplished through learning algorithms which try to mimic how the human brain learns.

Machine learning, which is a field that had grown out of the field of artificial intelligence, is of utmost importance as it enables the machines to gain human like intelligence without explicit programming.

However AI programs do the more interesting things such as web search or photo tagging or email anti-spam. So, machine learning was developed as a new capability for computers and today it touches many segments of industry and basic science. There is autonomous robotics, computational biology. Around $90 \%$ of the data in the world was generated in the last two years itself and the inclusion of machine learning library known as Mahout into Hadoop ecosystem has enabled to encounter the challenges of Big Data, especially unstructured data.

In the area of machine learning research the emphasis is given more on choosing or developing an algorithm and conducting experiments on the basis of the algorithm. Such highly biased view reduces the impact or real world applications.

In this paper the various applications under the appropriate category of machine learning has been highlighted. This paper makes an effort to bring all the major areas of applications under one umbrella and present a more general and realistic view of the real world applications. Apart from this two application suggestions have been presented forward. The field of machine learning is so vast and ever growing that it proves to be useful in automating every facet of life.

\section{MACHINE LEARNING}

According to Arthur Samuel Machine learning is defined as the field of study that gives computers the ability to learn without being explicitly programmed. Arthur Samuel was famous for his checkers playing program.

Initially when he developed the checkers playing program, Arthur was better than the program. But over time the checkers playing program learned what were the good board positions and what were bad board positions are by playing many games against itself.

A more formal definition was given by Tom Mitchell as a computer program is said to learn from experience (E) with respect to some task $(\mathrm{T})$ and some performance measure $(\mathrm{P})$, if its performance on $\mathrm{T}$, as measured by $\mathrm{P}$, improves with experience $\mathrm{E}$ then the program is called a machine learning program.

In the checkers playing example the experience $\mathrm{E}$, was the experience of having the program playing games against itself. The task $T$ was the task of playing checkers. And the performance measure $\mathrm{P}$, was the probability that it won the next game of checkers against some new opponent.

In all fields of engineering, there are larger and larger data sets that are being understood using learning algorithms.

\section{TYPES OF MACHINE LEARNING ALGORITHMS}

\subsection{Supervised Learning}

This learning process is based on the comparison of computed output and expected output, that is learning refers to computing the error and adjusting the error for achieving 
the expected output. For example a data set of houses of particular size with actual prices is given, then the supervised algorithm is to produce more of these right answers such as for new house what would be the price.

\subsection{Unsupervised Learning}

Unsupervised learning is termed as learned by its own by discovering and adopting, based on the input pattern. In this learning the data are divided into different clusters and hence the learning is called a clustering algorithm. One example where clustering is used is in Google News (URL news.google.com). Google News groups new stories on the web and puts them into collective news stories.

\subsection{Reinforcement Learning}

Reinforcement learning is based on output with how an agent ought to take actions in an environment so as to maximize some notion of long-term reward. A reward is given for correct output and a penalty for wrong output. Reinforcement learning differs from the supervised learning problem in that correct input/output pairs are never presented, nor sub-optimal actions explicitly corrected.

\subsection{Recommender Systems}

Recommender systems can be defined as a learning techniques by virtue of which online user can customize their sites to meet customer's tastes. For example, online user can get a rating of a product or/ and related items when he/she searching an items because of the existing recommender system. That is why it changed the way people find products, information, and even other people. There are mainly two approaches: content based recommendation and collaborative recommendation, which help the user for obtaining and mining data, making intelligent and novel recommendations, ethics. Most e-commerce site uses this system. [58]

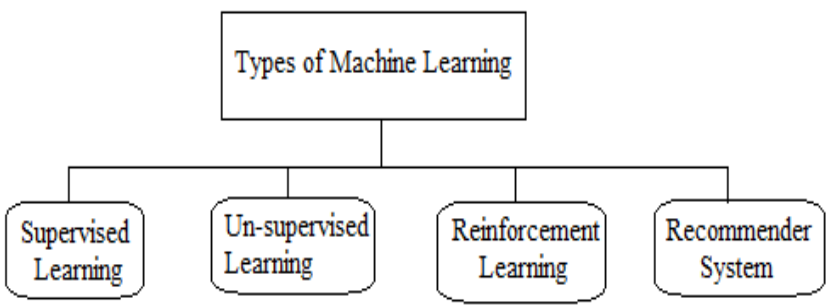

Figure-2: Types of Machine Learning

\section{APPLICATIONS OF MACHINE LEARNING AND LITERATURE SURVEY}

This section elaborates classified applications of machine learning according to different machine learning algorithm under supervised learning, unsupervised learning, reinforcement learning and recommender learning.

\subsection{Unsupervised Learning}

In machine learning, the problem of unsupervised learning is that of trying to find hidden structure in unlabeled data. Since the examples given to the learner are unlabeled, there is no error or reward signal to evaluate a potential solution.

\subsubsection{DNA classification: Understanding genomics}

Figure 3 shows a DNA microarray data, the colors, red, green, gray and so on, show the degree to which different individuals do or do not have a specific gene. The idea is to form a group of different individuals such that each of them has a certain gene. So a clustering algorithm can be run to group individuals into different categories or into different types of people. So this is Unsupervised Learning because the algorithm is not given any information in advance whether there are type 1 people, type 2 persons, and type 3 persons and so on. Instead a bunch of data is given and the algorithm automatically finds structure in the data into these types of individuals. [23]

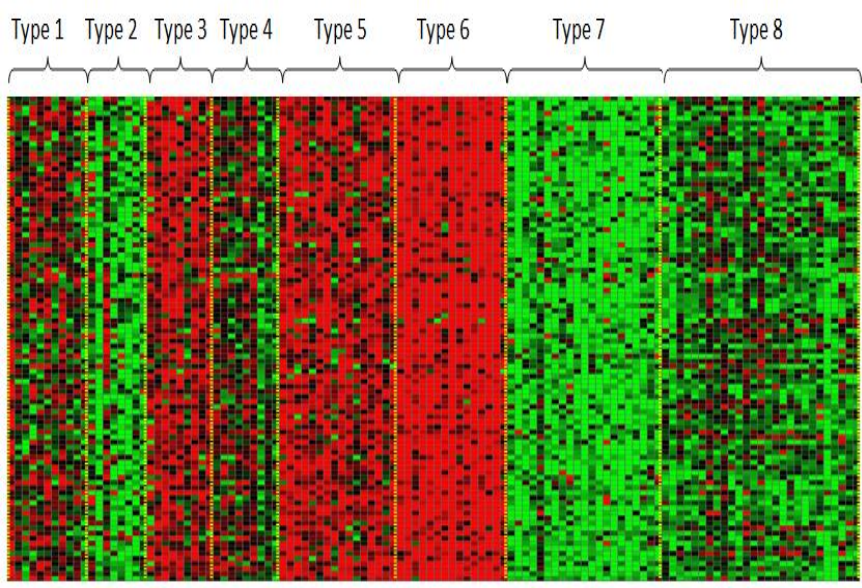

Figure 3: DNA microarray data

\subsubsection{Organizing large computer clusters:}

At large data centers that are large computer clusters, unsupervised learning helps to figure out which machines tend to work together, so that if those machines are put together or if there is some crisis, then the data centers can work more efficiently. [16]

\subsubsection{Social network analysis:}

Unsupervised Machine learning algorithms can automatically identify the friends within a user circle in Facebook or Google, or it can identify the maximum number of mails sent to a particular person and categorize into collective groups. It also identifies which are groups of people that all know each other. [17]

\subsubsection{Market segmentation:}

Many companies have huge databases of customer information. So, Unsupervised Machine learning algorithms can look at this customer data set and automatically discover market segments and automatically group customers into different market segments so that the company can automatically and more efficiently sell or market the different market segments together. Again, this is Unsupervised Learning because it is not known in advance what the market segments are, or which customer belongs to which segment [18]

\subsubsection{Astronomical data:}

\subsubsection{Astronomical data analysis:}

These clustering algorithms give surprisingly interesting useful theories of how galaxies are born.

\subsubsection{Anomaly/Novelty detection in astronomical data:}

Modern astronomical observatories are very advanced and can produce massive amount of data which the researchers don't even have time to look at. Sometimes the researchers even lack the adequate knowledge, experience and training to 
deduce the exact significance or meaning of these data sets. It is not unusual that these large-scale astronomical data sets can contain anomalies/novelties. Thus the need for machines which can be trained to go through the data generated and in the process detect any anomalies that may be present in the data set (at a much faster rate and in most cases with better accuracy) becomes evident. Anomaly/Novelty Detection is the process of finding unusual things or characteristics which are different from our prevalent knowledge about the data. Anomalies detection problems are primarily of two types: 1 ) point anomaly - anomalies of this kind are individual celestial objects that present unusual characteristics. 2) group anomalies - this is an unusual collection of points. A group of points can be considered abnormal either because it is a collection of anomalous points, or because that the way its member points aggregate is unusual, even if the points themselves are perfectly normal. [30]

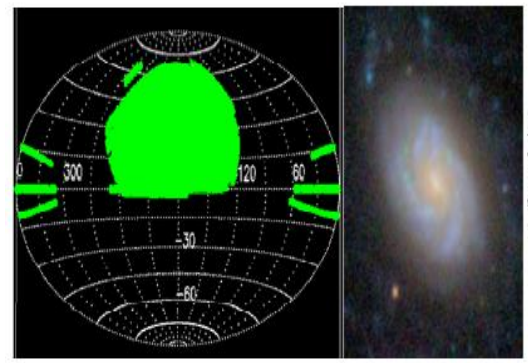

(a)

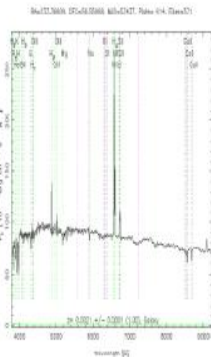

(c)
Figure 4: Summary of the Sloan Digital Sky Survey (SDSS) data set. (a) The coverage map of SDSS. (b) A sample imaging data. (c) A sample spectroscopic data i.e. the spectrum.

\subsubsection{The cocktail party problem:}

At a cocktail party with two people, two people talking at the same time. Two microphones are put in the room at two different distances from the speakers; each microphone records a different combination of these two speaker voices. Maybe speaker one is a little louder in microphone one and maybe speaker two is a little bit louder on microphone two because the 2 microphones are at different positions relative to the 2 speakers, but each microphone would cause an overlapping combination of both speakers' voices. These two microphone recorders are given to an Unsupervised Learning algorithm called the cocktail party algorithm. The cocktail party algorithm separates out these two audio sources that were being added or being summed together. [19]

\subsubsection{Medical records:}

With the advent of automation, electronic medical records have become prevalent, so if medical records are turned into medical knowledge, then disease could be understood in a better way. [21] [35]

\subsubsection{Computational biology:}

Computational biology also known as bioinformatics is the use of biological data to develop algorithms and establish relations among various biological systems. With automation again, biologists are collecting lots of data about gene sequences, DNA sequences, gene expression array analysis, combinatorial chemistry and so on, and machines running algorithms are providing a much better understanding of the human genome, and what it means to be human. [22] [31] [32] [45] [50]

\subsubsection{Analysis of gene expression data: cancer diagnosis:}

Cancer can be defined as a class of diseases that is characterized with out of control cell growth. There are about a 100 different types of cancer claiming the lives of innumerable people across the world. Thus identifying the type of cancer is a crucial step in its treatment. It is done through classification of patient samples. The classification process and results may be improved by analysing the gene expression of the patient which may provide additional information to the doctors. The merger of medical science and technology has already led to a lot of life saving breakthroughs in the field of medicine. Thus the involvement of technology in fighting cancer is of no surprise. Machine learning techniques, such as Bayesian networks, neural trees, and radial basis function (RBF) networks, are used for the analysis of the datasets and classifying cancer types. These techniques have their own properties including the ability of finding important genes for cancer classification, revealing relationships among genes, and classifying cancer. [33] [34] [40] [48]

\subsubsection{Speech Activity Detection (SAD):}

Power of speech is a primary way for humans to express themselves. Often the audio or speech contains silent pauses which are pauses where speech is absent; this is where speech activity detection (SAD) finds its application. SAD is a technique used to detect the presence of human speech, it can help reduce the load on human listeners by removing long and noisy non-speech intervals. SAD is language independent and can be of two types namely: Supervised and Unsupervised. Supervised SAD depends largely on the training data so its use is limited to the availability of training data and consistency of the test environment whereas Unsupervised SAD is a feature-based technique where performance degrades with increase in noise. Speech activity detection (SAD) has applications in a variety of contexts such as speech coding, automatic speech recognition (ASR), speaker and language identification, and speech enhancement. [53]

\subsubsection{Acoustic Factor Analysis for Robust Speaker Verification:}

Identification or recognition of the speaker by analysing the voice data for authentication is Speaker Recognition or Verification. Mismatch between training and test conditions represent one of the most challenging problems facing researchers in this field today. Some of the sources of introduction of these mismatches are: transmission channel differences, handset variability, background noise, and session variability due to physical stress, vocal effort such as whisper, Lombard effect, non-stationary environment, and spontaneity of speech. In order to enable machines to produce reliable and authenticate data researchers need to train them to eliminate or overcome these mismatches. One of the ways in which this can be achieved is the analysis of the acoustic factors which are supposed to represent the listener's efficiency in processing directional cues, while suppressing some unwanted channel components. [55]

\subsection{Supervised Learning}

Supervised learning is the machine learning task of inferring a function from labeled training data. The training data consist of a set of training examples. In supervised learning, each example is a pair consisting of an input object (typically a vector) and a desired output value. A supervised learning algorithm analyzes the training data and produces an inferred 
function, which can be used for mapping new examples.

\subsubsection{E-mail data:}

\subsubsection{Automatic answering of incoming} messages:

Instead of typing out the same reply every time someone emails with a common queries and problems, now machine learning algorithms analyses those mails and automatically generates a reply. This proves useful in case of large companies. [1]

\subsubsection{Automatic mail organization into folders:}

With the bulk amount of messages pouring daily it proves highly inconvenient for users to segregate the messages manually. Therefore machine learning proves to be most beneficial by categorizing the mail automatically into various user-defined inbox tabs such as primary, social, promotions, update, forums etc. If a particular message from a particular sender is moved from update tab to primary tab, then all other future messages from that user will end up in the primary tab. [1]

\subsubsection{Email and thread summarization:}

The incoming messages are analyzed and the important sentences are extracted from the email thread and are composed into a summary. This summary is generated based on special characteristics of email. [1]

\subsubsection{Spam filtering:}

It is mainly used to filter unsolicited bulk Email (UBE), junk mail, or unsolicited commercial email (UCE) from the genuine e-mails. The spam filter saves the user from having to wade through tons of spam email, that's also a learning algorithm. The spam filter can also be learned by watching which emails you do or do not flag as spam. So in an email client if spam button is clicked to report some email as spam, but not other emails and based on which emails are marked as spam, the e-mail program learns better how to filter spam e-mail. [1] [29]

\subsubsection{Email Batch Detection:}

The problem of detecting batches of emails that have been created according to the same template needs to be addressed. This problem is motivated by the desire to filter spam more effectively by exploiting collective information about entire batches of jointly generated messages. Senders of spam, phishing, and virus emails avoid mailing multiple identical copies of their messages. Once a message is known to be malicious, all subsequent identical copies of the message could be blocked easily, and without any risk of erroneously blocking regular emails. [27]

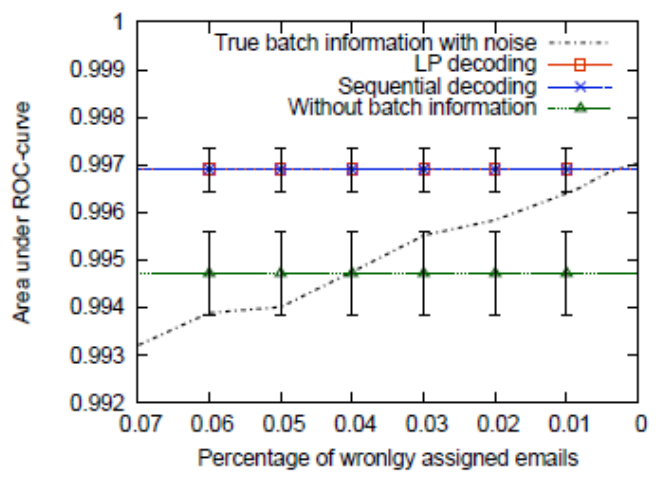

Figure 5: Classification accuracy with batch information

\subsubsection{Handwriting recognition:}

It turns out one of the reasons it's so inexpensive today to route a piece of mail across the countries, is that when an address is written on an envelope, it turns out there's a learning algorithm that has learned how to read the handwriting so that it can automatically route this envelope on its way, and so it costs less. [4]

\subsubsection{Face recognition:}

Human face is not unique, rigid object and numerous factors cause the appearance of the face to vary. There are numerous application areas where face recognition can be exploited such as security measure at an ATM, areas of surveillance, closed circuit cameras, image database investigation, criminal justice system, and image tagging in social networking sites like Face book etc. [5]

\subsubsection{Speech recognition:}

All speech recognition software utilizes machine learning. Speech recognition systems involve two distinct learning phases: one before the software is shipped (training the general system in a speaker-independent fashion), and a second phase after the user purchases the software (to achieve greater accuracy by training in a speaker -dependent fashion). [3]

\subsubsection{Information retrieval:}

Information retrieval (IR) is finding material (usually documents) of an unstructured nature (usually text) that satisfies an information need from within large collections (usually stored on computers). The user provides an outline of their requirements - perhaps a list of keywords relating to the topic in question, or even an example document. The system searches its database for documents that are related to the user's query, and presents those that are most relevant. The information retrieval process can be divided into four distinct phases: indexing, querying, comparison, and feedback. All phases of information retrieval can be performed manually, but automation has many benefitslarger document collections can be processed more quickly and consistently, and new techniques can be easily implemented and tested. The instant availability of enormous amounts of textual information on the Internet and in digital libraries has provoked a new interest in software agents that act on behalf of users, sifting through what is there to identify documents that may be relevant to users' individual needs. [10]

\subsubsection{Operating system:}

One of the main purposes of using computers is to get the job done as fast as possible. In such a scenario it is important that the applications start and respond quickly thus reducing he waiting time for the user. Different computer users have different usage preferences, which mostly refer to applications that are used most frequently by the user. This fact can be used by the underlying operating system for predicting the user application choices and pre-fetching them into the local memory for speedy start-up. This is achieved with the help of inbuilt software which trains itself by observing the actions of the user over time and learning from them. The Super-Fetch subsystem present in the kernel of Microsoft's Windows Vista operating system is an example of such a system. [2]

\subsubsection{Natural language processing or computer vision:}

These are the fields of AI pertaining to understanding 
language or understanding images. Most of natural language processing and most of computer vision today is applied machine learning.

\subsubsection{Intrusion detection:}

Intrusion detection is the process of monitoring the events that are occurring in the systems or networks and analyzing them for signs of possible incidents, which are violations or threats to computer security policies, acceptable use policies, or standard security practices It is mainly of two types based on the intrusions first is Misuse or signature based detection and the other is Anomaly detection. [11]

\subsubsection{Anomaly detection or recognizing anomalies:}

Detection of unusual sequences of credit card transactions, detection of unusual patterns of sensor reading in a nuclear power plant or unusual sound in car engine for such purpose dynamic machine learning method is used where instead of looking at individual operation, a sequence of operations are analyzed as a whole so that it is more robust to minor shifts in legitimate behavior. [12]

\subsubsection{Signature based detection:}

This technique of detection looks for evidence which indicates misuse. In a network, predetermined attack patterns forms a signature and these signatures are used to determine further network attacks .Machine learning enables examination of the network traffic with predefined signatures and each time database is updated. An example of Signature based Intrusion Detection System is SNORT. [13]

\subsubsection{Epileptic Seizure Detection:}

Epilepsy is a central nervous system disorder, in which the patient suffers from recurrent seizures that occur at unpredictable times and usually without warning. Seizures can result in a lapse of attention or a whole-body convulsion. Frequent seizures increase an individual's risk of sustaining physical injuries and may even result in death. With the help of supervised learning we can to construct patient-specific detectors capable of detecting seizure onsets quickly and with high accuracy. These classifiers detect the onset of an epileptic seizure through analysis of the scalp electroencephalogram (EEG), a non-invasive measure of the brain's electrical activity. [25]

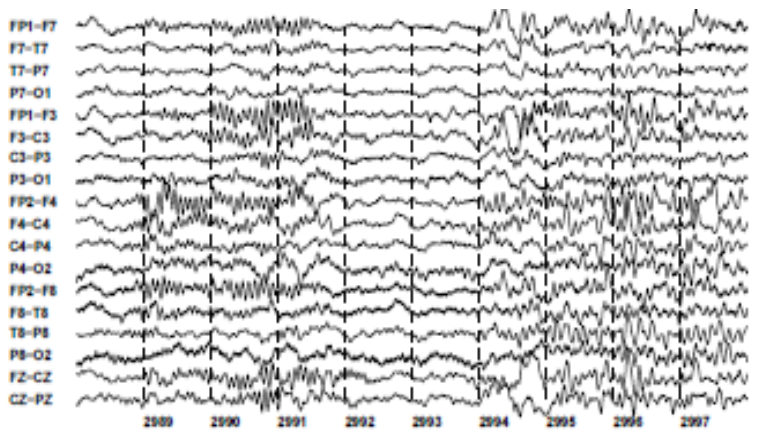

Figure 6: A seizure within the scalp EEG of a patient

\subsubsection{Automated Text Categorization:}

In document categorization, the texts are catalogued and it is a problem in library science, information science and computer science. The task is to assign a document to one or more classes. This may be done algorithmically. The documents to be classified may be texts, images, music, etc. Each kind of document possesses its special classification problems. When not otherwise specified, text classification is implied. Text categorization (TC-a.k.a. text classification, or topic spotting), the activity of labelling natural language texts with thematic categories from a predefined set, is one such task. TC is now being applied in many contexts, ranging from document indexing based on a controlled vocabulary, to document filtering, automated metadata generation, word sense disambiguation, etc. According to the machine learning (ML) paradigm a general inductive process automatically builds an automatic text classifier by learning, from a set of preclassified documents, the characteristics of the categories of interest. [28] [29] [31].

\subsubsection{Automatic Indexing for Boolean Information Retrieval Systems:}

Here each document is assigned one or more key words or key phrases describing its content, where these key words and key phrases belong to a finite set called controlled dictionary. [28]

\subsubsection{Document Organization:}

Indexing with a controlled vocabulary is an instance of the general problem of document base organization. [28]

\subsubsection{Text Filtering:}

Text filtering is the activity of classifying a stream of incoming documents dispatched in an asynchronous way by an information producer to an information consumer. [28]

\subsubsection{Word Sense Disambiguation:}

Word sense disambiguation (WSD) is the activity of finding, given the occurrence in a text of an ambiguous word, the sense of this particular word occurrence. [28]

\subsubsection{Hierarchical Categorization of Web Pages:}

Automatic classification of Web pages, or sites, under hierarchical catalogues.[28]

\subsubsection{Data Center Optimization:}

The modern data center (DC) is a complex interaction of multiple mechanical, electrical and controls systems. The sheer number of possible operating configurations and nonlinear interdependencies make it difficult to understand and optimize energy efficiency. One of the most complex challenges is power management. Growing energy costs and environmental responsibility have placed the DC industry under increasing pressure to improve its operational efficiency. The application of machine learning algorithms to existing monitoring data provides an opportunity to significantly improve DC operating efficiency. A typical large-scale DC generates millions of data points across thousands of sensors every day, yet this data is rarely used for applications other than monitoring purposes. Advances in processing power and monitoring capabilities create a large opportunity for machine learning to guide best practice and improve DC efficiency. The objective is to provide a data driven approach for optimizing DC performance. Neural network is selected as the mathematical framework for training DC energy efficiency models. Neural networks are a class of machine learning algorithms that mimic cognitive behaviour via interactions between artificial neurons. They are advantageous for modelling intricate systems as they search for patterns and interactions between features to automatically generate a best fit model. As with most learning systems, the model accuracy improves over time as new training data is acquired. [26] 


\subsubsection{SVM and Dimensionality Reduction in Cognitive Radio:}

With the passing time machine learning is gaining popularity and finding usage in various fields. Cognitive radio is one such field where machine learning finds its application. Cognitive radio is a radio that allows dynamic programming options and is designed to provide more wireless communication by detecting and using the best wireless channels available in a given area. The control of degrees of freedom (DOF) i.e. dimensionality reduction is considered to be the initial phase for radar and sensing signal processing. Whereas, SVM or Support Vector Machines are models that use a learning algorithm for developing pattern recognition and classification capabilities. These two approaches i.e. dimensionality reduction and SVM can be applied jointly to Cognitive Radio thus obtaining performance enhancements while classifying wireless signal data. The reason behind this gain in performance measure is that application of dimensionality reduction helps in doing away with the redundant signal data thus improving classification by the SVM method. [32] [41] [42] [43]

\subsubsection{Classification of Software Engineering Artifacts Using Machine Learning:}

A large amount of data is produced during the course of development of Software projects. The data generated in the process is not only vast in its quantity but also varying in the nature of its contents; it can include a range of different type of information pieces like the deployment details of the software system, component analysis, object and class models etc; not only this, but the interrelations among these information documents provide further insights to the project. It is natural that each one of these artifacts has some distinguishing attributes which can be used to categorize the data and hence make them more manageable and put them to constructive uses. The problem to this approach is that classifying such huge amount data which is ever on the increase is no task for humans this is where machine learning comes in. Machine learning can be used to develop a network which uses the defining properties of the existing artifacts for training itself in the task of classification and then carry on with the task of categorizing the artifacts by itself. [36]

\subsubsection{Computational Finance:}

In today's world the financial market is one of the most unstable and unpredictable. One has to be on his feet constantly in order to survive and be successful in this market. In such an environment where market crashes and sustained periods of loss, are common phenomenon and techniques of machine learning have emerged as the leading performance measures used in the industry. For example systems have bee developed where the future stock prices moves can be predicted by training an automated intelligent agent that discover patterns in the stock prices dynamic right before a major market move. During the exploitation stage, the agent observes current state of market. If a pattern recognized that was seen before, agent gives a buy/sell signal. Examples of commonly considered features include market volatility, total volume and amount of open interest. [38] [44] [46] [47]

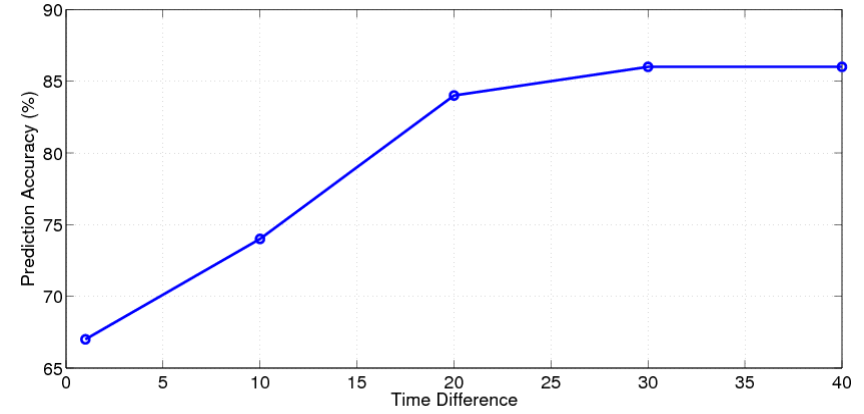

Figure 7: Accuracy of long-term prediction

\subsubsection{Semantic Scene Classification:}

In pattern recognition scene classification is a very common task in which the system scans a picture and analyzes the various elements in it and uses them to categorize the image to a certain class or group. In this process often such a situation may occur when the classes are not mutually exclusive by definition. For example a semantic scene classification, categorizes images into semantic classes such as beaches, sunsets or parties, in which many images may belong to multiple semantic classes. Figure 8 shows an image that falls under both beach and an urban scene categories. This multiple classification however is not ambiguous as one would think but is fully a member of each class (due to multiplicity). The same can occur in other types of categorisation like text or music categorization, medical diagnosis, etc. Multi-label machine learning provides methods that treats such cases differently and offers a solution to the challenges posed by them to the classic pattern recognition paradigm. [39]

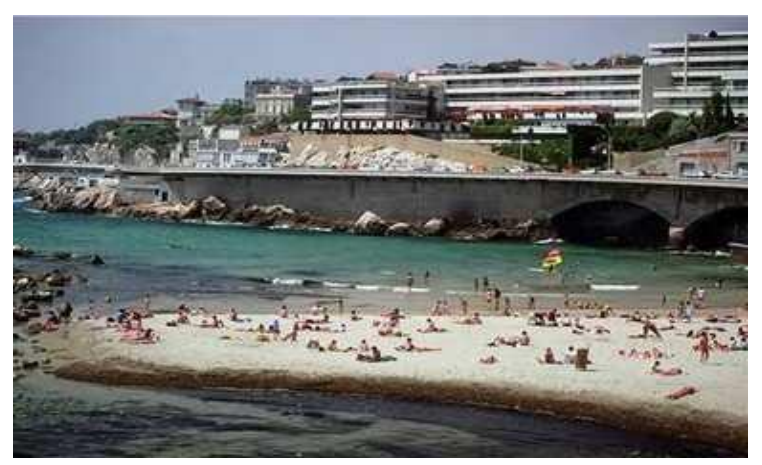

Figure 8: Image that falls under both beach and an urban scene categories

\subsubsection{Applications to music:}

Music is a vast sphere. The amount of data and material available here is huge and almost every individual hosts a different taste from others when it comes to music. Naturally the need for classification arises. One can classify music in a number of ways as there exists an ocean of options to select from when it comes to choosing a feature on whose basis the classification is to be done. Musical data is complex and often highly dimensional (when represented as audio) and this is where machines come to our assistance as machine learning is very well suited for working with such data. Classification is just one of the innumerable tasks that can be executed on such a data set as music using machine learning. Among other tasks we have music genre classification, music transcription, instrument classification, beat detection, blind instrument separation, capturing musical features, such as melody, harmony and rhythm to name a few. With the digitalization of music, a new and rapidly growing research 
area has emerged, called Music Information Retrieval (MIR) which is a research focused on the extraction of information from music audio and musical scores. Music Production is another relatively new field of research in this context. With machine learning we are able to alleviate some of the distance between musician and machine. Neural networks can be applied both to music audio signals and MIDI (Music Instrument Digital Interface) data. For example a neural network can be trained with the user's music rating history and time stamps and then it can be used to select songs more suitable to the user's activities during a day. [51]

\subsubsection{Evolving Signal Processing for Brain- Computer Interfaces (BCI):}

A BCI or Brain Computer Interface is collaboration between a brain and a device or machine that reads the electrical signals from the brain the uses them to guide some external activities like moving the cursor or a prosthetic limb. The device or machine acts as an interface between the brain and the object to be controlled by the brain. Figure 9 shows a conceptual schematic overview of evolving BCI design principles. Data obtained from sensors and devices within, on, and around a human subject are transformed into informative representations via domain-specific signal preprocessing. The resulting signals are combined to produce psychomotor state representations. These estimates may be made available to the systems the subject is interacting with. [52]

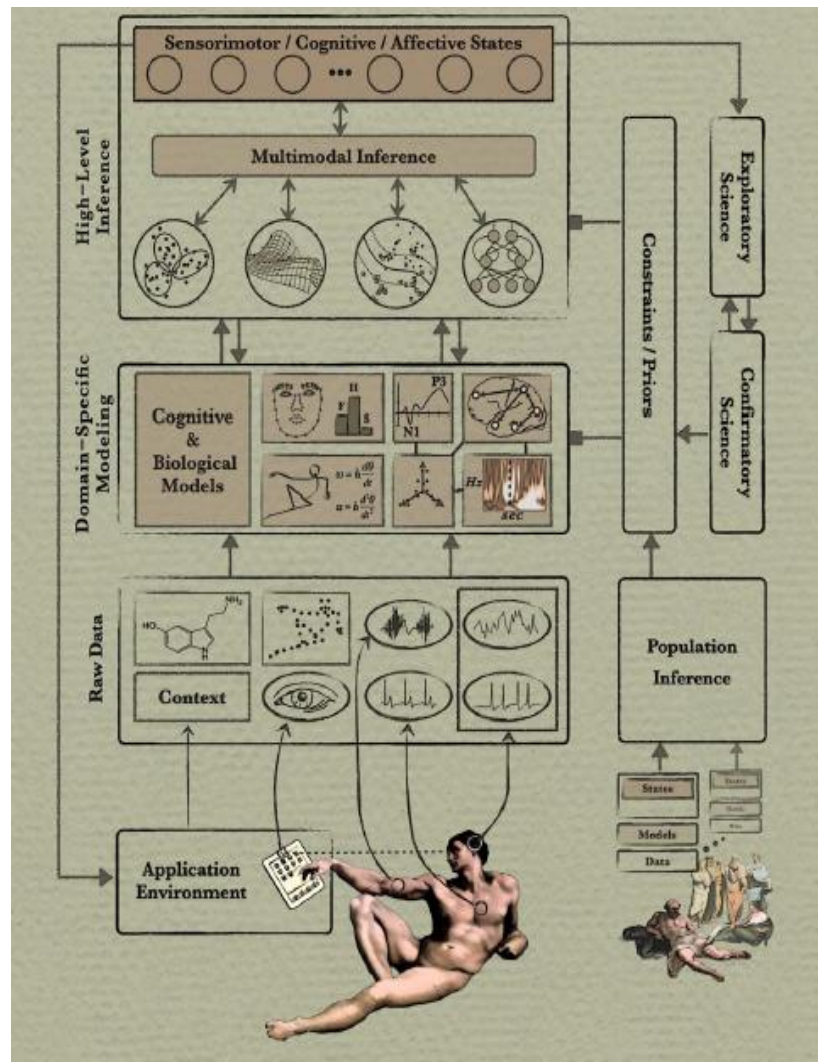

Figure 9: Conceptual schematic overview of evolving BCI design principles

\subsubsection{Acoustic Environment Identification (AEI) and Audio Forensics.}

An audio recording are prone to a number of possible distortions and artifacts like acoustic reverberation, background noise, etc. These disturbances which are otherwise considered to be useless and are attributed to degrading the quality of the audio can be put to advantage and used to extricate important information via various techniques like Acoustic Environment Identification (AEI), Audio Forensics, and ballistic settings. Of these, the acoustic environment identification (AEI) has a wide range of applications ranging from audio recording integrity authentication to real-time crime localization/identification whereas forensic audio enhancement can be used to reveal subtle or idiosyncratic background sounds that could provide important investigative clues. [54]

\subsection{Recommender system}

Recommender systems are a subclass of information-retrieval system that seeks to predict the 'rating' or 'preference' that user would give to an item, which allow the online customer to choose the best item.

\subsubsection{Mobile Learning Environments:}

Mobile learning (m-learning) means "learning on the move" which differs from typical e-learning where there is wastage of bandwidth [6]. Information can be easily accessed as and when desired due to the mobile or portable devices. So machine learning caters the learning process of different users by providing information which is customized to the preferences of the user. [7]

\subsubsection{Computational advertisement:}

Computational advertisement is a new scientific subdiscipline, at the intersection of Large scale search and text analysis, Information retrieval, Statistical modeling, Machine learning, Classification, Optimization, Microeconomic, Recommender systems. Computational advertisement is almost the exact opposite of classical advertisement which has, billions of opportunities, billions of creativities, totally personalize-able, tiny cost per opportunity and much more quantifiable. Computational advertisement intends to find the "best match" between a given user in a given context and a suitable advertisement. The context could be a user entering a query in a search engine ("sponsored search"), a user reading a web page ("content match" and "display ads") a user watching a movie on a portable device, and so on. [9]

\subsubsection{Sentiment analysis/ opinion analysis:}

When we hear a person speak we hear the words as well as the emotions in the person's voice and if the conversation is face to face we see their expressions as well. Textual data captures the facts and information but it mostly fails to capture the sentiments of the speaker leading to the misinterpretation of the true essence of the words. This can be seen as a loss of valuable information. Hence, sentiment or overall opinion towards the subject matters - for example, whether a product review is positive or negative. However sentiment analysis can be challenging and it must update itself with the ever complex use of statements to express and so learning algorithm proves to be significantly beneficial to that effect. Sentiment classification proves to be helpful in business intelligence applications, movie reviews and recommender systems. [15]

\subsubsection{Database mining (DM):}

With the growth of the web and automation came much larger data sets than ever before. In such a scenario an important task is to maintain these data in such a way that can prove to be useful. Effective algorithms need to be developed that can use this data to learn and serve the users more efficiently. For example, tons of Silicon Valley companies are today collecting web click data, also called 
click stream data, and are trying to use machine learning algorithms to mine this data to understand and serve the users better. [20] [37] [49]

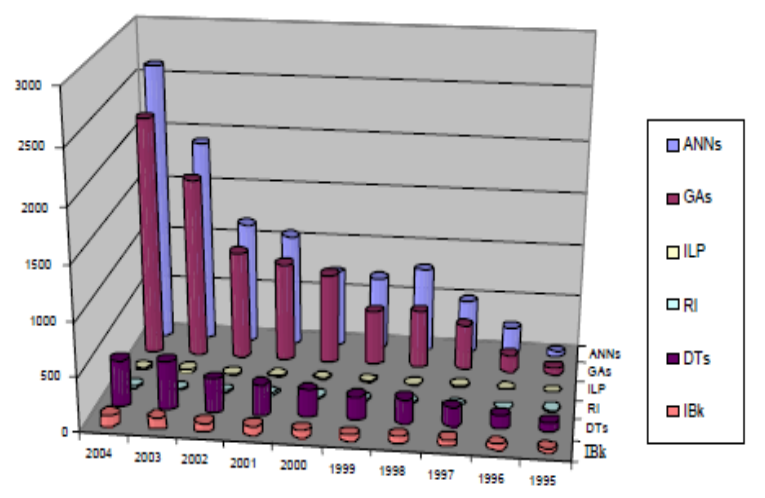

Figure 10: Comparative results on ML techniques applied to DM tasks over the period 1995-2004.

\subsubsection{Self-customizing programs:}

Today we resort to the internet in order to meet a host of our needs like listening to music and watching videos online, downloading songs, movies, apps etc, shopping, banking, making reservations, making travelling arrangements and so on. It is common experience for users to get recommendations from the sites that they visit based on their activity history on that site. The sites achieve this by means of a learning algorithm which learns by observing the user activities and choices over time and customizes itself to the users' preferences. Learning algorithms are being used today to understand human learning and to understand the brain.

\subsection{Reinforcement learning}

Reinforcement learning is an area of machine learning inspired by behaviorist psychology, concerned with how software agents ought to take actions in an environment so as to maximize some notion of cumulative reward.

\subsubsection{Traffic forecasting service:}

With the ever increasing number of vehicles plying on the roads traffic management seems to a huge problem these days. Machines can be trained and used to solve this problem. For example, systems that overlay predictions about future traffic conditions on a digital traffic flow map. These systems can also be used to know the current and future traffic conditions of a region and also provide users with routing options based on that information.[57]

\subsubsection{Computer games:}

The gaming industry has grown tremendously in the recent years. AI driven agents are used widely to create interactive gaming experience for the players. These agents can take a variety of roles such as player's opponents, teammates or other non-player characters. Apart from interacting with the human players, a game needs to satisfy a host of other requirements like the audio and visual effects, the gaming environment etc the different fields of machine learning caters to all these needs and helps programmers develop games that are well suited to the present market demands. [8]

\subsubsection{Machinery applications:}

There are those applications that cannot be programmed by hand. For example, autonomous helicopters in which the computer could learn by itself on how to fly the helicopters.

\subsubsection{Stock market analysis:}

The stock market and its trends keep changing day in and day out and in order to be able to make profit and survive in this financial market proper understanding of it and prediction skills are must haves. Although many lack that insight and the task is tedious and keeps getting difficult with the evolution of the business world, the obvious solution to this is computers. Machine learning has been extensively used for prediction of financial markets. Popular algorithms, such as support vector machine (SVM) and reinforcement learning have been quite effective in tracing the stock market and maximizing the profit of stock option purchase while keeping the risk low. It also incorporates sentiment analysis which considers the opinions of the general investors in addition to that the global stock data is included to predict the next-day stock trend. [14]

\subsubsection{Semantic Annotation of Ubiquitous Learning Environments:}

In today's world practical knowledge is gaining importance fast in almost every field. It not only helps in acquiring practical skills which are more helpful on-field but also gives better understanding of the subject to the person studying it. Moreover evaluation of skill- based learning systems helps researchers to better understand how students are learning The use of semantic annotations as part of a skills-based learning environment is very useful in this case. Simulations of real life situations help in the promotion and of practical skills like decision making, team working, communication, and problem solving. They can be incorporated in the process of assessment of students' performance. The University of Southampton for example has such a clinical skills laboratory; Figure 11. The ward contains computerized and interactive simulated mannequins, non-computerized mannequins, and a range of equipments which provides clinical set-up and activities for the students. The students are given a number of tasks to perform and the computerized mannequins are programmed to alter their parameters to a point of significant deterioration in health such that emergency responses would be required from the students. These activities provoke the students to perform as they would in a real situation such as move themselves around the ward, to interact with each other and the supervising staff members, etc. As the students and mentors are "immersed" in the simulation and behaving "as in real practice," the captured video data can be used to provide important information about their performance. Skills-based helps ensure that practitioners are "fit for practice". [56]

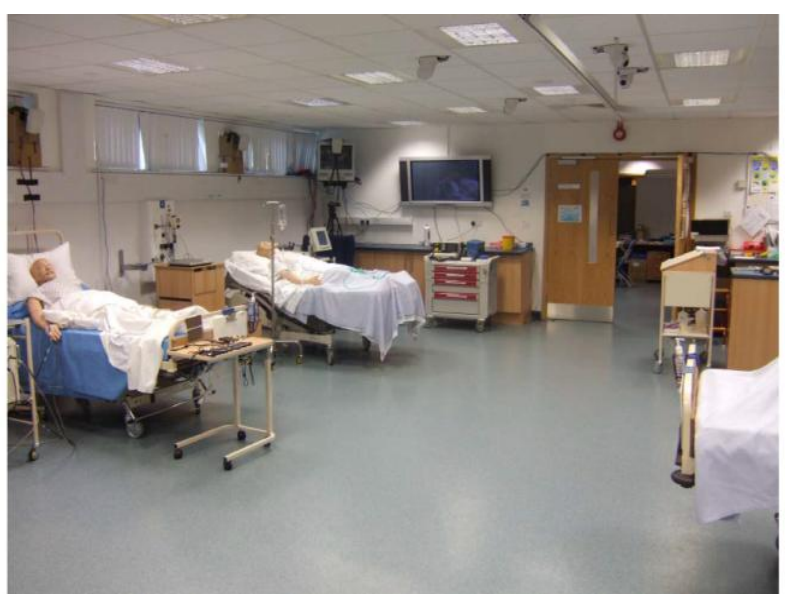

Figure 11 Clinical skills laboratory 


\section{IMPRESSION AND VIEWS}

With the amount of data set getting large by every passing day, the analysis of these immense amounts of data is beyond the capacity of human eye. So Artificial agents take up the responsibility of interacting with the environment and in turn influence it. The inception of the "Big Data" has also resulted in improvisation of the machine learning algorithms as they have larger data sets to gain more experience. The concern is not how big "Big Data" is but it's more about finding patterns within it.

In Machine learning the artificial agents learns from training data or by interacting with the environment and influences it to facilitate the best possible result. So Machine Learning is definitely a subfield of Artificial Intelligence. This notion has made the present day applications autonomous.

In the field of medicine and diagnosis AI has created virtual doctors as shown in Figure-12. Providing the early symptoms to a machine algorithm helps in early detection and diagnosis of the disease. The ultimate desire is to create a diagnostic dream machine for this purpose. [35] [50]

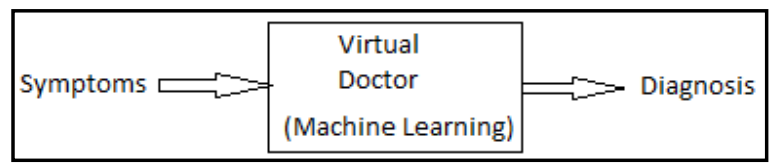

Figure-12: Virtual Doctor

In the context of search engine, machine learning not only provides result on the basis of the search content but also gives preferences to the users' choices and activity online, which has resulted in a complete revolution of the search engines.

Machine learning can prove immensely helpful in the process of building an information time machine as shown in Figure14. Information time machine requires large database of the present and the past. One of the ways to extrapolate the database of the past is to digitize the historical archives in which case machine learning can prove useful.

$\begin{aligned} & \text { Historical } \\ & \text { Archives }\end{aligned}=$ Machine Learning $=-\begin{gathered}\text { Digitised } \\ \text { Information }\end{gathered}$

\section{Figure-13 Information Time Machine}

The best result so far has been the invention of autonomous driving vehicles making use of Machine learning, making the routers more intelligent in a network and also application in cloud computing is a big prospect.

As in Machine learning, supervised and unsupervised learning are of the two major types. And AI agents are general problem solvers and can be applied in various fields.

So, AI is not about perfectly replicating human, it's about figuring out the principles that allow agents to act intelligently and improving upon us. The bottom line is that intelligence is no longer exclusive to only humans.

\section{CONCLUSION}

Humans have always sought to build a comfortable life, the proof of this lies in the fact that we have always depended on machines to get our work done more easily, in a faster and more efficient manner. In the past machines have been used to reduce the manual labor required get a job done, but at present, with the advent of machine learning humans seek to build machines which are not only strong but also intelligent and hence machine learning has emerged to become an area of study that is ever in the bloom. Machine learning has not just made the machines autonomous, bringing forward the concept of autonomous computing, but it has also reduced the constant vigilance users are required to keep upon the applications. In this paper, discusses the four categories of machine learning i.e. supervised learning, unsupervised learning, and reinforcement learning and recommender system and also presents the numerous applications under them. Apart from that two proposed applications namely information time machine and virtual doctor have been put forward. The main purpose of machine learning is to develop algorithms that assist in the creation of intelligent machines thus reducing the jobs of the programmers as the machine learns in due course of time to improve its performance. Although a lot of advancements have been made in this field still then there exists glaring limitations in the data set from which machine learns. It can be rectified by constantly keeping the data sets up-to-date as learning is a continuous process. Apart from this issue, a great number of publications on machine learning evaluate new algorithms on a handful of isolated benchmark data sets. In spite of all these shortcomings machine learning has solved varying problems of global impact. Machine learning has proven to be vastly useful in a variety of fields such as data mining, artificial intelligence, OCR, statistics, computer vision, mathematical optimization, etc and its importance tends to remain ever on the increase. Machine learning theories and algorithm are inspired from the biological learning systems where the performance depends on factors like the amount of available data, the learning history and experience, etc, and thus help explaining human learning. The applications of machine learning are therefore never ending and it still remains an active field of research with immense development options and a promising future.

Future challenge is to develop emergence automated prescription at critical condition using machine learning concept, which can minimize the error in diagnosis.

\section{ACKNOWLEDGMENTS}

Our thanks to the experts Dr. Susanta Biswas, Kalyani University, who have advised and encouraged us for such kind of development. Also our special thanks to $\mathrm{Dr}$ Somsubhra Gupta, JIS College of Engineering for providing all kinds of required resources.

\section{REFERENCES}

[1] Tzanis, George, et al. "Modern Applications of Machine Learning." Proceedings of the 1st Annual SEERC Doctoral Student Conference-DSC. 2006.

[2] Horvitz, Eric. "Machine learning, reasoning, and intelligence in daily life: Directions and challenges." Proceedings of. Vol. 360. 2006.

[3] Mitchell, Tom Michael. The discipline of machine learning. Carnegie Mellon University, School of Computer Science, Machine Learning Department, 2006.

[4] Ball, Gregory R., and Sargur N. Srihari. "Semisupervised learning for handwriting recognition." Document Analysis and Recognition, 2009. ICDAR'09. 10th International Conference on. IEEE, 2009.

[5] Valenti, Roberto, et al. "Machine learning techniques for face analysis." Machine Learning Techniques for 
Multimedia. Springer Berlin Heidelberg, 2008. 159-187.

[6] Al-Hmouz, Ahmed. "An adaptive framework to provide personalisation for mobile learners." (2012).

[7] Al-Hmouz, Ahmed, Jun Shen, and Jun Yan. "A machine learning based framework for adaptive mobile learning." Advances in Web Based Learning-ICWL 2009. Springer Berlin Heidelberg, 2009. 34-43.

[8] Thore Graepel "Playing Machines: Machine Learning Applications in Computer Games", ICML 2008 Tutorial - 5 July 2008, Helsinki, Finland.

[9] Broder, Andrei, and Vanja Josifovski. "Introduction to computational advertising." (2010).

[10] Cunningham, Sally Jo, James Littin, and Ian H. Witten. "Applications of machine learning in information retrieval." (1997).

[11] Kaur, Harjinder, Gurpreet Singh, and Jaspreet Minhas. "A Review of Machine Learning based Anomaly Detection Techniques." arXiv preprint arXiv:1307.7286 (2013)

[12] Wiese, Bénard, and Christian Omlin. Credit card transactions, fraud detection, and machine learning: Modelling time with LSTM recurrent neural networks. Springer Berlin Heidelberg, 2009.

[13] Kumar, Vinod, and Dr Om Prakash Sangwan. "Signature Based Intrusion Detection System Using SNORT." International Journal of Computer Applications \& Information Technology 1 (2012)

[14] Shen, Shunrong, Haomiao Jiang, and Tongda Zhang. "Stock market forecasting using machine learning algorithms." (2012).

[15] Pang, Bo, Lillian Lee, and Shivakumar Vaithyanathan. "Thumbs up?: sentiment classification using machine learning techniques." Proceedings of the ACL-02 conference on Empirical methods in natural language processing-Volume 10. Association for Computational Linguistics, 2002.

[16] Liao, Shih-wei, et al. "Machine learning-based prefetch optimization for data center applications." Proceedings of the Conference on High Performance Computing Networking, Storage and Analysis. ACM, 2009.

[17] Haider, Peter, Luca Chiarandini, and Ulf Brefeld. "Discriminative clustering for market segmentation." Proceedings of the 18th ACM SIGKDD international conference on Knowledge discovery and data mining. ACM, 2012.

[18] Haider, Peter, Luca Chiarandini, and Ulf Brefeld. "Discriminative clustering for market segmentation." Proceedings of the 18th ACM SIGKDD international conference on Knowledge discovery and data mining. ACM, 2012.

[19] Haykin, Simon, and Zhe Chen. "The cocktail party problem." Neural computation 17.9 (2005): 1875-1902.

[20] Clarke, Bertrand, Ernest Fokoue, and Hao Helen Zhang. Principles and theory for data mining and machine learning. Springer Science \& Business Media, 2009.

[21] Kononenko, Igor. "Machine learning for medical diagnosis: history, state of the art and perspective." Artificial Intelligence in medicine 23.1 (2001): 89-109.
[22] Caragea, Cornelia, and Vasant Honavar. "Machine Learning in Computational Biology." Encyclopedia of Database Systems (2009): 1663-1667.

[23] Cho, Sung-Bae, and Hong-Hee Won. "Machine learning in DNA microarray analysis for cancer classification." Proceedings of the First Asia-Pacific bioinformatics conference on Bioinformatics 2003-Volume 19 Australian Computer Society, Inc., 2003.

[24] Wagstaff, Kiri. "Machine learning that matters." arXiv preprint arXiv:1206.4656 (2012).

[25] Shoeb, Ali H., and John V. Guttag. "Application of machine learning to epileptic seizure detection." Proceedings of the 27th International Conference on Machine Learning (ICML-10). 2010.

[26] Gao, Jim, and Ratnesh Jamidar. "Machine Learning Applications for Data Center Optimization." Google White Paper (2014)

[27] Haider, Peter, Ulf Brefeld, and Tobias Scheffer. "Supervised clustering of streaming data for email batch detection." Proceedings of the 24th international conference on Machine learning. ACM, 2007.

[28] Sebastiani, Fabrizio. "Machine learning in automated text categorization." ACM computing surveys (CSUR) 34.1 (2002): 1-47.

[29] Bratko, Andrej, et al. "Spam filtering using statistical data compression models." The Journal of Machine Learning Research 7 (2006): 2673-2698.

[30] Xiong, Liang, et al. "Anomaly detection for astronomical data." (2010).

[31] Guyon, Isabelle, and André Elisseeff. "An introduction to variable and feature selection." The Journal of Machine Learning Research 3 (2003): 1157-1182.

[32] Hou, Shujie, et al. "SVM and Dimensionality Reduction in Cognitive Radio with Experimental Validation." arXiv preprint arXiv:1106.2325 (2011).

[33] Hwang, Kyu-Baek, et al. "Applying machine learning techniques to analysis of gene expression data: cancer diagnosis." Methods of Microarray Data Analysis. Springer US, 2002. 167-182.

[34] Luca Silvestrin, "Machine Learning in Biology", Universita degli studi di Padova.

[35] Magoulas, George D., and Andriana Prentza. "Machine learning in medical applications." Machine Learning and its applications. Springer Berlin Heidelberg, 2001. 300-307.

[36] Bruegge, Bernd, et al. "Classification of Software Engineering Artifacts Using Machine Learning."

[37] Shhab, Areej, Gongde Guo, and Daniel Neagu. "A Study on Applications of Machine Learning Techniques in Data Mining." Proc. of the 22nd BNCOD workshop on Data Mining and Knowledge Discovery in Databases, Sunderland, UK. 2005.

[38] Boyarshinov, Victor. Machine learning in computational finance. Diss. Rensselaer Polytechnic Institute, 2005.

[39] Shen, Xipeng, et al. "Multilabel machine learning and its application to semantic scene classification." Electronic Imaging 2004. International Society for 
Optics and Photonics, 2003

[40] Zararsiz, Gokmen, Ferhan Elmali, and Ahmet Ozturk. "Bagging Support Vector Machines for Leukemia Classification." development 1 (2012): 2.

[41] Tsagkaris, Kostas, Apostolos Katidiotis, and Panagiotis Demestichas. "Neural network-based learning schemes for cognitive radio systems." Computer Communications 31.14 (2008): 3394-3404.

[42] Tabaković, Željko. "A Survey of Cognitive Radio Systems." Post and Electronic Communications Agency, Jurišićeva 13

[43] Hosey, Neil, et al. "Q-Learning for Cognitive Radios." Proceedings of the China-Ireland Information and Communications Technology Conference (CIICT 2009). ISBN 9780901519672. National University of Ireland Maynooth, 2009.

[44] Pawar, Prashant. Machine Learning applications in financial markets. Diss. Indian Institute of Technology, Bombay Mumbai.

[45] Tarca, Adi L., et al. "Machine learning and its applications to biology." PLoS computational biology 3.6 (2007): e116.

[46] Prof. St'ephan $\mathrm{Cl}^{\prime}$ emen ,“A Machine-Learning View of Quantitative Finance”, con - Institut Mines Telecom LTCI UMR Telecom Paris Tech.

[47] Shen, Shunrong, Haomiao Jiang, and Tongda Zhang. "Stock market forecasting using machine learning algorithms." (2012).

[48] Wang, Yu, et al. "Gene selection from microarray data for cancer classification - a machine learning approach." Computational biology and chemistry 29.1 (2005): $37-$ 46.

[49] Prof. Pier Luca Lanzi, Laurea in Ingegneria Informatica, Politecnico di Milano, Polo di Milano Leonardo,"Machine Learning, Data Mining, and Knowledge Discovery: An Introduction"

[50] Sajda, Paul. "Machine learning for detection and diagnosis of disease." Annu. Rev. Biomed. Eng. 8 (2006): 537-565.

[51] Øland, Anders. "Machine Learning and its Applications to Music." (2011).
[52] Makeig, S.; Kothe, C.; Mullen, T.; Shamlo, N. B.; Zhang, Z. \& Kreutz-Delgado, K. (2012), 'Evolving Signal Processing for Brain-Computer Interfaces.', Proceedings of the IEEE 100 (Centennial-Issue) , 15671584 .

[53] Seyed Omid Sadjadi and John H. L. Hansen, "Unsupervised Speech Activity Detection Using Voicing Measures and Perceptual Spectral Flux", IEEE signal processing letters, March 2013.

[54] Hafiz Malik, "Acoustic Environment Identification and Its Applications to Audio Forensics". IEEE Transactions on Information Forensics and Security, Vol. 8, No. 11, November 2013.

[55] "Acoustic Factor Analysis For Robust Speaker Verification, Fellow, IEEE. IEEE Transactions On Audio, Speech, And Language Processing, Vol. 21, No 4, April 2013.

[56] Mark J. Weal, Danius T. Michaelides, Kevin Page, David C. De Roure, Fellow, IEEE, Eloise Monger, and Mary Gobbi. , "Semantic Annotation of Ubiquitous Learning", Environments IEEE Transactions On Learning Technologies, Vol. 5, No. 2, April-June 2012.

[57] EJ Horvitz, J Apacible, R Sarin, L Liao - arXiv preprint arXiv:1207.1352, 2012 - arxiv.org

[58] https://www.coursera.org/learn/recommender-systems

\section{AUTHOR'S PROFILE}

Sumit Das is presently working as an Asst. Professor in the Department of Information Technology, JIS College of Engineering, West Bengal, India. He completed his M.Tech degree in Computer Science and Engineering from University of Kalyani in the year 2008.

Akash Pal is a student of 4th year in Computer Science \& Engineering Department in JIS College of Engineering, West Bengal, India.

Aritra Dey is a student of 3rd year in Electrical Engineering Department in JIS College of Engineering, West Bengal, India.

Nabamita Roy is a student of 4th year in Computer Science \& Engineering Department in JIS College of Engineering, West Bengal, India 